\title{
A Utilisation of Improved Gabor Transform for Harmonic Signals Detection and Classification Analysis
}

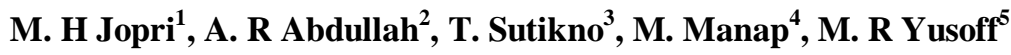 \\ ${ }^{1,2}$ Faculty of Electrical Engineering, Universiti Teknikal Malaysia Melaka, Malacca, Malaysia \\ ${ }^{3}$ Department of Electrical Engineering, Universitas Ahmad Dahlan, Yogyakarta, Indonesia \\ ${ }^{4,5}$ Faculty of Engineering Technology, Universiti Teknikal Malaysia Melaka, Malacca, Malaysia
}

\section{Article Info}

Article history:

Received Oct 19, 2016

Revised Dec 21, 2016

Accepted Jan 11, 2017

\section{Keyword:}

Classification analysis

Gabor transform

Harmonic signal

Power distribution system

Signal detection

Time-frequency distributions

\begin{abstract}
This paper presents a utilization of improved Gabor transform for harmonic signals detection and classification analysis in power distribution system. The Gabor transform is one of time frequency distribution technique with a capability of representing signals in jointly time-frequency domain and known as time frequency representation (TFR). The estimation of spectral information can be obtained from TFR in order to identify the characteristics of the signals. The detection and classification of harmonic signals for 100 unique signals with numerous characteristic of harmonics with support of rule-based classifier and threshold setting that been referred to IEEE standard 1159 2009. The accuracy of proposed method is determined by using MAPE and the outcome demonstrate that the method gives high accuracy of harmonic signals classification. Additionally, Gabor transform also gives 100 percent correct classification of harmonic signals. It is verified that the proposed method is accurate and cost efficient in detecting and classifying harmonic signals in distribution system.
\end{abstract}

Copyright $@ 2017$ Institute of Advanced Engineering and Science. All rights reserved.

\section{Corresponding Author:}

M.H Jopri,

Advanced Digital Signal Processing Group,

Faculty of Electrical Engineering,

Universiti Teknikal Malaysia Melaka,

Hang Tuah Jaya, 76100 Durian Tunggal, Melaka, Malaysia.

Email: hatta@utem.edu.my

\section{INTRODUCTION}

Power quality (PQ) monitoring has become an important part of power distribution system in order to prevent equipment damage and to determine the root cause of the power quality disturbances (PQDs) [1]. One of the crucial issues in power quality (PQ) is harmonic and inter-harmonics disturbance. As indicated by (IEEE Std. 1159:2009), harmonics which caused by rectifiers and inverters, are the inverse of interharmonics where they are frequency components of distorted voltages and currents that are integer multiples of the fundamental frequency $(50 \mathrm{~Hz}$ for Malaysia). Harmonic combines with the fundamental voltage or current produces waveform distortion. Harmonic distortion exists due to the nonlinear characteristics of devices and loads on the power system. Meanwhile, inter-harmonic is a frequency components of distorted voltage that are not integer multiples of the fundamental frequency and can leads to excitation of low frequency mechanical oscillations and malfunction in ripple control [2]. Numerous methods are developed and among these methods, signal processing (SP) technique has received intensified attention from the researchers and utility engineers [3]. The role of SP technique is to identify, classify and characterize the large volume of data, obtained using modern measuring instruments. The most familiar technique for frequency-domain analysis is the Fourier transform (FT). Nevertheless, it only works for stationary signal and it is not capable to resolve any signal associated with fluctuations. To resolve this, the STFT is 
introduced, whereby STFT divides the signal into small segments. Thus these signal segments can be assumed to be stationary. Moreover, the STFT works well providing that the window is short enough compared to the oscillation rate. Nevertheless, during high rates of oscillation, it can lead to significant errors [4]. Due to this limitation, the Discrete Fourier transform (DFT) is presented for analysis of frequency content in steady state and suitable for harmonic analysis. However, DFT is not capable to detect sudden changes in waveform. Furthermore, the DFT has major problems such as spectrum leakage and resolution [5]. The limitation in DFT is overcome by using Wavelet analysis. Wavelet analysis is an approach to vary the window length. Nevertheless, wavelet reveals some disadvantages such as its complex computation and sensitive to noise level [6]. With regards to the above discussion, the constraint of previous techniques can be overcome by introducing Gabor transform.

In this paper, the Gabor transform (GT) is one of time frequency distribution (TFD) technique and good in distinguishing the harmonic and inter-harmonic signals in power distribution system. The GT is the windowed Fourier transform and the representation of a signal in a jointly time-frequency domain [7]. The harmonic signals are analyzed and represented in time frequency representation (TFR). Furthermore, the parameters such as RMS and fundamental value, total harmonic distortion (THD), total non-harmonic distortion (TnHD) and total waveform distortion (TWD) for voltage and current are evaluated from TFR and used for the classification of harmonic and inter-harmonic signals. The performance of the proposed technique is verified by classifying 100 signals with numerous characteristics for every type of voltage variation signals and also based on IEEE Std. 1159-2009.

\section{RESEARCH METHOD}

Time-frequency distribution (TFD) are superior methods that represent signals in time and frequency representation (TFR). Furthermore, from TFR, spectral parameters can be seen with the changes of time. In this manner, the TFD are significant to classify harmonic and inter-harmonic signals in the system. Linear TFD which is Gabor transform is proposed as in Figure 1 for the signal classification technique.

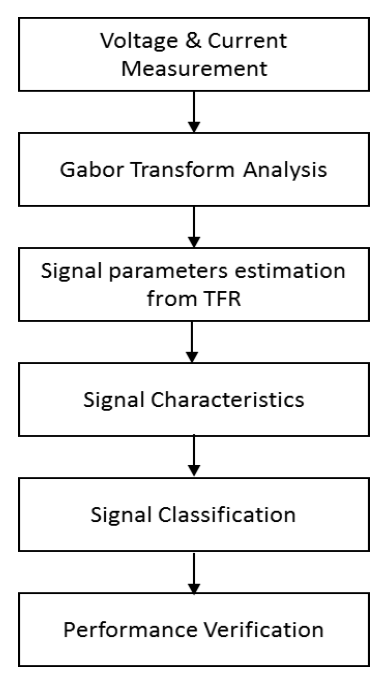

Figure 1. Implementation Chart for Harmonic and Inter-harmonic Signals Detection and Classification

\subsection{Gabor Transform}

Gabor transform (GT) is an expanding of signal into a set of functions that are concentrated in both the time and frequency domains and then use the coefficients as the descriptors of the signal's local property. Gabor transform is defined as [7].

$$
C(n, k)=\int_{-\infty}^{\infty} x(\tau) h^{*}(n, k) d \tau
$$

where $x(\tau)$ is the signal under analysis and $h(n, k)$ is the set of elementary function and is defined as 


$$
h(n, k)-w(\tau-\mathrm{nT} \omega) e^{-j 2 \pi k f o \tau}
$$

where $w(\tau)$ is the observation window. Tw and $\mathrm{f} 0$ are the time and frequency sampling interval, respectively, that must satisfy the Heisenberg uncertainty relationship as follows:

$$
T_{w} f_{0} \geq \frac{1}{4 \pi}
$$

For Gabor transform, hanning window is selected same as spectrogram but different in terms of window length which is 480 samples. The time and frequency resolution for Gabor transform is fixed for all frequency shown in Figure 2. The vertical axis (x-axis) shows the time resolution and horizontal axis (y-axis) shows the frequency resolution.

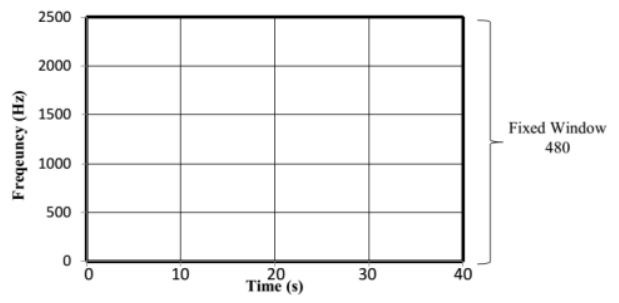

Figure 2. Resolution of Gabor Transform

\subsection{Signal Parameters}

Parameters of harmonic and inter-harmonic are assessed from the TFR. The spectral parameters consist of RMS fundamental voltage, momentary RMS voltage, instantaneous total inter-harmonic distortion (TnHD), instantaneous total harmonic distortion (THD) and instantaneous total waveform distortion (TWD). Below are the signal parameters that been estimated from TFR [8].

Instantaneous RMS Voltage, $V_{r m s}(t)=\sqrt{\int_{0}^{f_{s}} P_{x}(t, f) d f}$

Instantaneous RMS Fundamental Voltage, $V_{1 r m s}(t)=\sqrt{2 \int_{f_{l o}}^{f_{h i}} P_{x}(t, f) d f}$

Instantaneous Total Waveform Distortion, $T W D(t)=\frac{\sqrt{V_{r m s}(t)^{2}-V_{1 r m s}(t)^{2}}}{V_{1 r m s}(t)}$

Instantaneous Total Harmonic Distortion, $\operatorname{THD}(t)=\frac{\sqrt{\sum_{h=2}^{H} V_{h, r m s}(t)^{2}}}{V_{1 r m s}(t)}$

Instantaneous Total Nonharmonic Distortion, $\operatorname{Tn} H D(t)=\frac{\sqrt{V_{r m s}(t)^{2}-\sum_{h=0}^{H} V_{h, r m s}(t)^{2}}}{V_{1 r m s}(t)}$ 
where $\operatorname{Px}(t, f)$ is the TFR of a signal, $f_{s}$ is sampling frequency, $f_{0}$ is the fundamental frequency, $V_{\text {Irms }(t)}$ is instantaneous RMS fundamental voltage, $V_{r m s(t)}$ is instantaneous RMS voltage and $V_{h, r m s}(t)$ is RMS harmonic voltage. $f_{h i}=f_{0}+25 \mathrm{~Hz}, f_{l o}=f_{0}-25 \mathrm{~Hz}, 25 \mathrm{~Hz}$ is chosen for $f_{h i}$ and $f_{l o}$, it can represent the fundamental frequency value and use for calculate the value of the frequency element.

\subsection{Signal Characteristic}

The identification of signal characteristics is obtained from the calculated spectral parameters. Moreover, by utilizing the instantaneous RMS voltage, the signal properties for instance the average of RMS voltage can be computed utilizing Equation 9 [9]. The data of the signal characteristics are used as input for classifier to identify the harmonic and inter-harmonics signal.

$$
V_{r m s, a v e}=\frac{1}{T} \int_{0}^{T} V_{r m s}(t) d t
$$

Furthermore, total nonharmonic distortion, $\mathrm{TnHD}_{\mathrm{ave}}$ and average of total harmonic distortion, $\mathrm{THD}_{\text {ave }}$ can be computed from instantaneous total nonharmonic distortion, $\operatorname{TnHD}(\mathrm{t})$ and instantaneous total harmonic distortion, $\mathrm{THD}(\mathrm{t})$ individually.

$$
\begin{aligned}
& T H D_{\text {ave }}=\frac{1}{T} \int_{0}^{T} T H D(t) d t \\
& T n H D_{\text {ave }}=\frac{1}{T} \int_{0}^{T} \operatorname{Tn} H D(t) d t
\end{aligned}
$$

\subsection{Signal Classification}

The rule-based classifier will be utilized for signal classification and this classifier is a deterministic grouping technique that has been utilized as a part of genuine application [20]. The performance of classification is much dependent on the principles and threshold values. Since in this research, all previous information that been obtained from power quality signals gave good information, this classifier is suitable to be used for of signal characterization. The utilization of rule-based classifier agreeing below requirements [10].

$$
\begin{aligned}
& T H D_{\text {ave }}>T n H D_{\text {thres }} \text { and } T n H D_{\text {ave }}<T n H D_{\text {thres }} \\
& T n H D_{\text {ave }}>=T n H D_{\text {thres }} \text { and } T H D_{\text {ave }}<T n H D_{\text {thres }}
\end{aligned}
$$

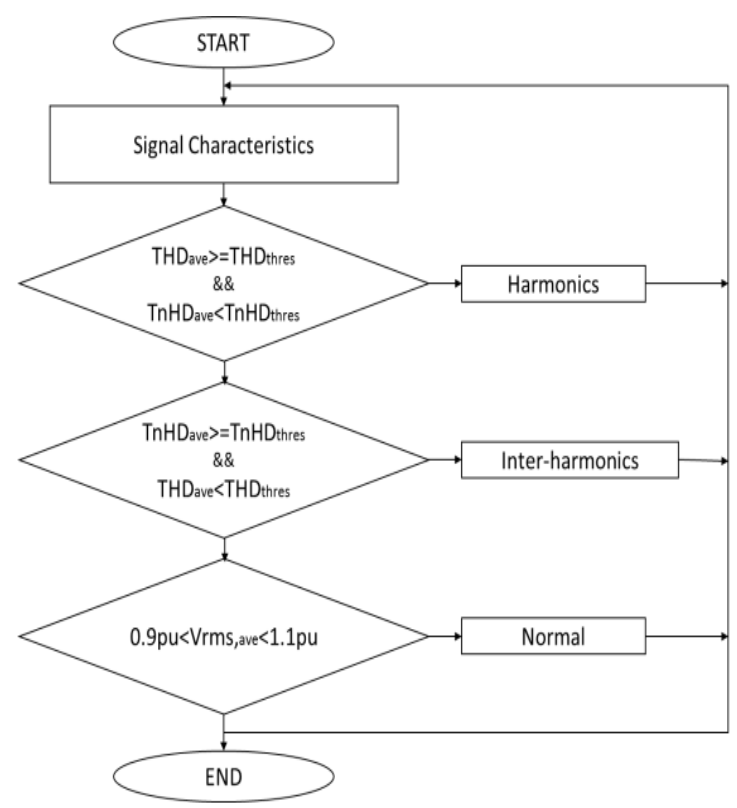

Figure 3. Rule-based Classifier Flow Chart 
In the meantime, flow chart of rule-based classifier for signal classification clearly shown in Figure $3 . \mathrm{THD}_{\text {thres }}$ and $\mathrm{TnHD}_{\text {thres }}$ are set according results of numerous investigation of these signals. The classification of signals can be done by observing the significant relationship concerning THD or TnHD. Hence, harmonic signal is classified when the signal has only THD whereas inter-harmonic signal has just TnHD.

\subsection{Performance Verification}

Keeping in mind the end goal to check the performance of proposed technique, the outcomes were assessed regarding accuracy and classification correctness during analysis. Subsequently, the execution and feasibility of proposed method relies on upon these said particulars.

\subsubsection{Accuracy of the Analysis}

The accuracy of the proposed technique is distinguished by measuring the measurement accuracy of signal characteristics. To quantify the accuracy of the outcome, mean absolute percentage error (MAPE) is utilized as benchmark and can be defined as [11],

$$
M A P E=\frac{1}{N} \sum_{n=1}^{N}\left|\frac{x_{i}(n)-x_{m}(n)}{x_{i}(n)}\right| x 100 \%
$$

where $x_{i}(n)$ is an actual value, $x_{m}(n)$ is measured value and $\mathrm{N}$ is the number of data. The smaller value of MAPE offers more accurate results.

\section{RESULTS AND ANALYSIS} discussion.

In this section, it is clarified the results of research and at the same time is given the comprehensive

\subsection{Harmonic Signals Detection}

Figure 4(a) and (b) present harmonic signal in time domain and its TFRs using Gabor transform, repectively. The TFR indicates that the signal consists of two frequency component: fundamental frequency $(50 \mathrm{~Hz})$ and 7 th harmonic component $(350 \mathrm{~Hz})$. Signal parameters estimated from the TFR using Gabor transform with OSWS are shown in Figure 4(b). Figure 4(c) shows that the harmonic voltage increases the RMS voltage from normal voltage which is 1.0 to $1.17 \mathrm{pu}$. However, it does not change the RMS fundamental voltage which remains constant at $1.0 \mathrm{pu}$. Besides that, the signal also results the magnitude of TWD and THD remains constant at $60 \%$ and zero percent for the TnHD as shown in Figure 4(d). Thus, the parameters clearly show that the harmonic signal consists only harmonic frequency components.

The results for interharmonic signal and its TFR using Gabor transform is shown in Figure 5(a) and (b) respectively. The signal has two frequency components which are at fundamental frequency $(50 \mathrm{~Hz})$ and interharmonic frequency of $375 \mathrm{~Hz}$. Signal parameters estimated from the TFR using Gabor transform with OSWS are shown in Figure 5(b). Figure 5(c) shows that the interharmonic voltage increases the RMS voltage from normal voltage which is 1.0 to $1.17 \mathrm{pu}$. However, it does not change the RMS fundamental voltage which remains constant at $1.0 \mathrm{pu}$. Besides that, the signal also results the magnitude of TWD and TnHD remains constant at $60 \%$ and zero percent for the THD as shown in Figure 5(d). Thus, the parameters clearly show that the interharmonic signal consists only nonharmonic frequency components. 


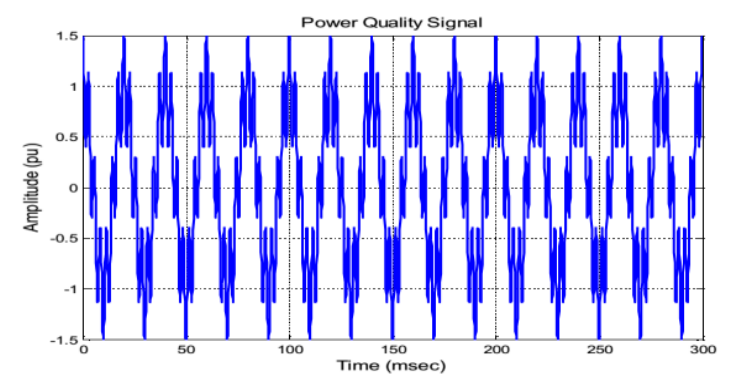

(a)
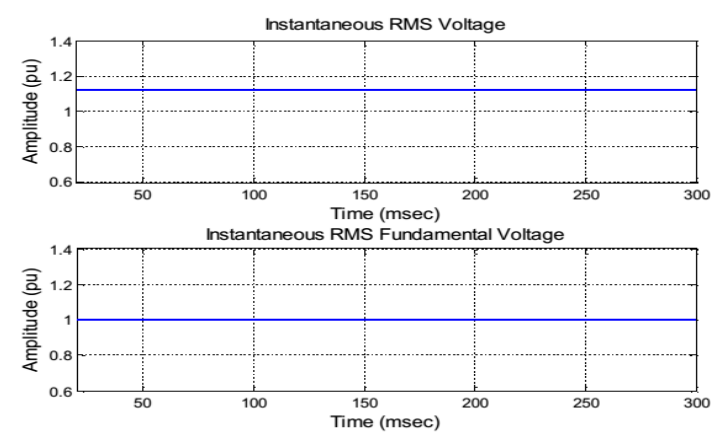

(c)

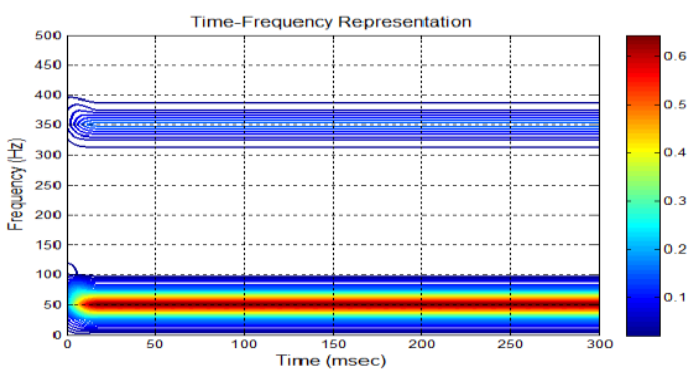

(b)
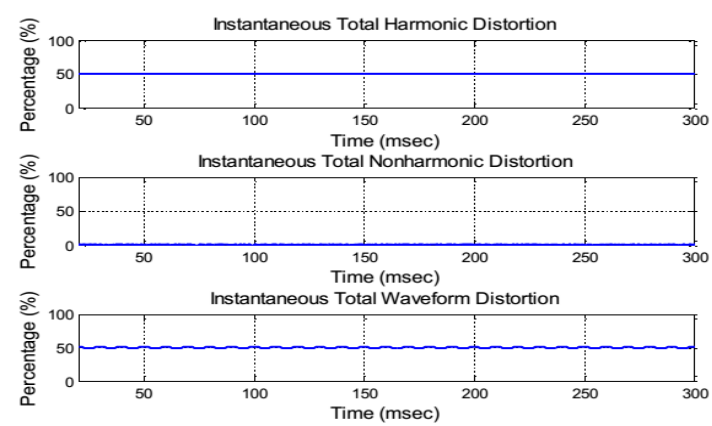

(d)

Figure 4. (a) Harmonic Signal from Simulation and its, (b) TFR using Gabor Transform, (c) Instantaneous of RMS and Fundamental RMS Voltage, (d) Instantaneous of total Harmonic Distortion, Total Nonharmonic Distortion and Total Waveform Distortion

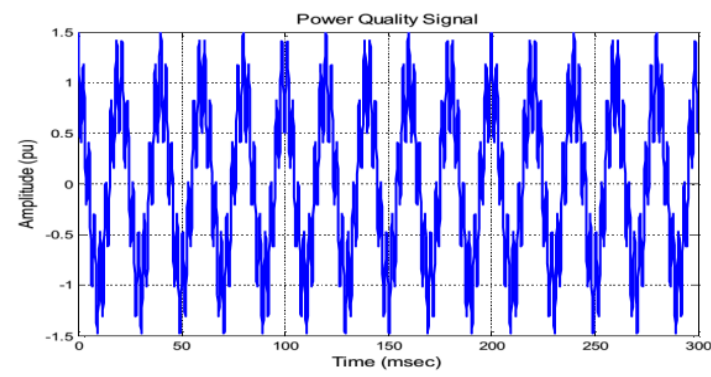

(a)
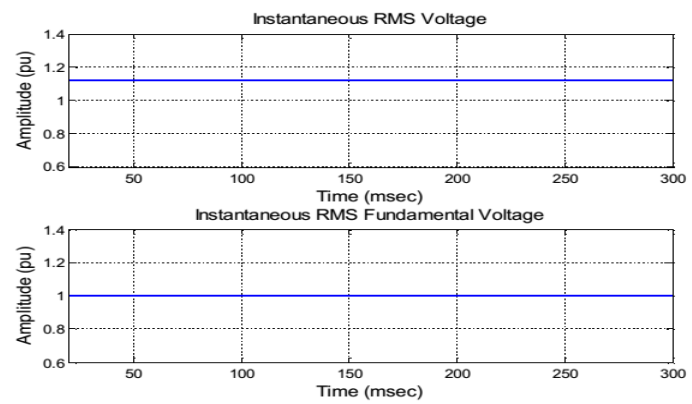

(c)

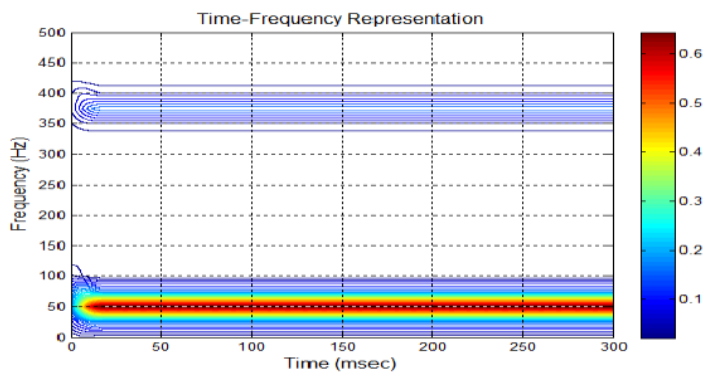

(b)

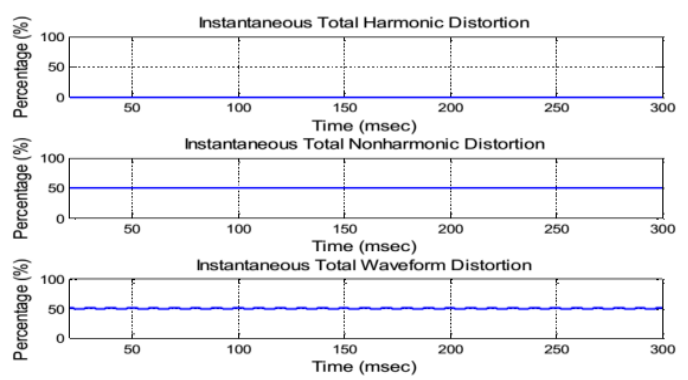

(d)

Figure 5. (a) Interharmonic Signal from Simulation and its, (b) TFR using Gabor Transform,

(c) Instantaneous of RMS and Fundamental RMS Voltage, (d) Instantaneous of Total Harmonic Distortion, Total Nonharmonic Distortion and Total Waveform Distortion 


\subsection{The accuracy of the Analysis}

Harmonic and inter-harmonics signals are tested and mean absolute percentage errors (MAPE) of the signal properties are calculated. Then, the results are averaged to identify the accuracy of the measurements as shown in Table 1. The table shows that Gabor transform gives good accuracy for an average of RMS voltage, THD and TnHD. In addition, Gabor transform has a good accuracy in harmonic and inter-harmonic signals detection.

Table 1. MAPE Simulation Result for Gabor Transform Analysis

\begin{tabular}{cc}
\hline Signal Characteristics & MAPE (\%) \\
\hline $\mathrm{V}_{\text {rms.ave }}$ & 0.5293 \\
THD $_{\text {ave }}$ & 0.9967 \\
TnHD $_{\text {ave }}$ & 0.9331 \\
\hline
\end{tabular}

\subsection{Classification of Harmonic and Inter-harmonic Signals}

As shown in the previous section, Gabor transform has less computational complexity and high accuracy. The performance results of the signals classification using the Gabor transform are shown in Table 2. 100 signals with various characteristics for each type of voltage signal is generated and classified. The table shows that the classification results using Gabor transform give $100 \%$ correct classification for all signals. From the results, it can be concluded that the Gabor-transform is magnificently a good method for harmonic and inter-harmonic signals classification.

Table 2. Performance of Harmonic and Inter-Harmonic Signals Classification

\begin{tabular}{ccc}
\hline Signal & \multicolumn{3}{c}{ Gabor Transform } \\
& Number of data sets & 100 \\
Harmonics & 100 & 100 \\
Inter-harmonics & 100 & 100 \\
Normal & 100 & Correct Classification \\
\hline
\end{tabular}

\section{CONCLUSION}

The performance evaluation of the signal analysis using Gabor transform in terms of accuracy and classification correctness have been shown clearly in results section. The performance of the proposed technique is verified by using MAPE in classifying 100 signals with various characteristics of voltage signals. The proposed method also gives 100 percent correctness of signals classification. Hence, it is concluded that the proposed method is a good technique for harmonic and inter-harmonic signals detection and classification in power distribution system.

\section{ACKNOWLEDGEMENTS}

This research is supported by Advance Digital Signal Processing Laboratory (ADSP Lab). Special thanks also to the Faculty of Electrical Engineering and Engineering Technology of Universiti Teknikal Malaysia Melaka (UTeM), Ministry of Higher Education Malaysia (MOHE) and Ministry of Science, Technology and Innovation (MOSTI) for giving the cooperation and funding for this research with grant number 06-01-14-SF00119 L00025. Their support is gratefully acknowledged.

\section{REFERENCES}

[1] M. S. Manikandan, et al., "Detection and Classification of Power Quality Disturbances Using Sparse Signal Decomposition on Hybrid Dictionaries," IEEE Trans. Instrum. Meas., vol/issue: 64(1), pp. 27-38, 2015.

[2] N. H. H. Abidullah, et al., "Real-Time Power Quality Disturbances Detection and Classification System," World Appl. Sci. J., vol/issue: 32(8), pp. 1637-1651, 2014.

[3] M. Gupta, et al., "Neural Network Based Indexing and Recognition of Power Quality Disturbances," TELKOMNIKA (Telecommunication Comput. Electron. Control., vol/issue: 9(2), pp. 227-236, 2011.

[4] F. Choong, et al., "Advances in Signal Processing and Artificial Intelligence Technologies in the Classification of Power Quality Events: A Survey,” Electr. Power Components Syst., vol/issue: 33(12), pp. 1333-1349, 2005.

[5] S. A. Deokar and L. M. Waghmare, "Integrated DWT-FFT approach for detection and classification of power quality disturbances,” Int. J. Electr. Power Energy Syst., vol. 61, pp. 594-605, 2014.

[6] R. Kumar, et al., "Recognition of Power Quality Events Using S- Transform Based ANN Classifier and Rule Based Decision Tree," 2013 IEEE Ind. Appl. Soc. Annu. Meet., pp. 1-8, 2013. 
[7] L. Debnath and F. A. Shah, "The Gabor Transform and Time-Frequency Signal Analysis," in Wavelet Transforms and Their Applications, Boston, MA: Birkhäuser Boston, pp. 243-286, 2015.

[8] A. R. Abdullah, et al., "Power Quality Monitoring System Utilizing Periodogram And Spectrogram Analysis Techniques," Int. Conf. Control. Instrum. Mechatronics Eng., no. August, pp. 770-774, 2007.

[9] N. A. Abidullah, et al., "Real-time power quality signals monitoring system," Proceeding - 2013 IEEE Student Conf. Res. Dev. SCOReD 2013, no. December, pp. 433-438, 2015.

[10] A. R. Abdullah, et al., "Performance Evaluation of Real Power Quality Disturbances Analysis Using S-Transform," Appl. Mech. Mater., vol. 752-753, pp. 1343-1348, 2015.

[11] K. R. Cheepati and T. N. Prasad, "Performance Comparison of Short Term Load Forecasting Techniques," Int. J. Grid Distrib. Comput., vol/issue: 9(4), pp. 287-302, 2016.

\section{BIOGRAPHIES OF AUTHORS}

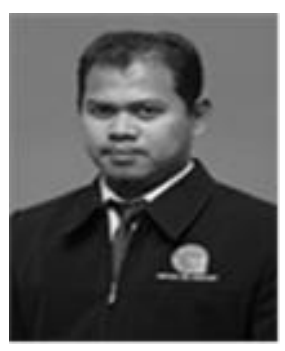

Mohd Hatta Jopri was born in Johor, Malaysia on 1978. He received his B.Sc from Universiti Teknologi Malaysia in 2000 and Msc. in Electrical Power Engineering from RheinischWestfälische Technische Hochschule Aachen (RWTH), Germany in 2011. Since 2005, he has been an academia staff in the Universiti Teknikal Malaysia Melaka (UTeM) and currently he pursuing his $\mathrm{PhD}$ in the area of power quality.

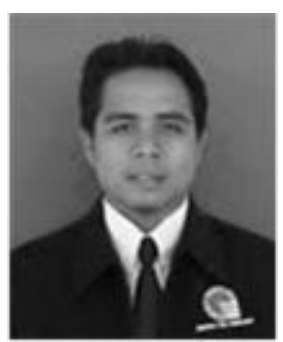

Dr. Abdul Rahim Abdullah was born in Kedah, Malaysia on 1979. He received his B. Eng., Master Eng., PhD Degree from Universiti Teknologi Malaysia in 2001, 2004 and 2011 in Electrical Engineering and Digital Signal Processing respectively. He is currently an Associate Professor with the Department of Electrical Engineering, Chief of Advanced Digital Signal Processing (ADSP) Lab and Center of Excellent (COE) Coordinator for Universiti Teknikal Malaysia Melaka (UTeM).

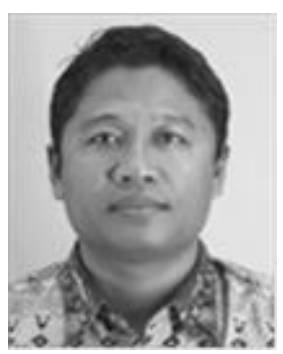

Tole Sutikno is an Associated Professor in Department of Electrical Engineering at Universitas Ahmad Dahlan (UAD), Indonesia. He is Editor-in-Chief, Principle Contact and Editor for some Scopus indexed journals. He is also one of founder of the Indonesian Publication Index (IPI). He has completed his studies for B.Eng., M.Eng., Ph.D. in Electrical Engineering from Diponegoro University, Gadjah Mada University and Universiti Teknologi Malaysia, respectively. His research interests include the field of industrial electronics and informatics, power electronics, FPGA applications, embedded systems, data mining, information technology and digital library.

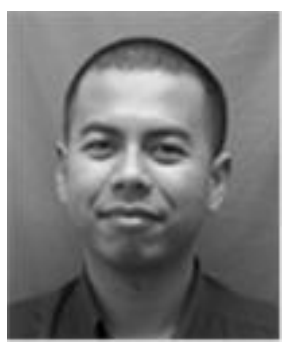

Mustafa Manap was born in Kuala Lumpur, Malaysia on 1978. He received his B.Sc from Universiti Technologi Malaysia in 2000 and Msc. in Electrical Engineering from Universiti Teknikal Malaysia Melaka (UTeM) 2016. Since 2006, he has been an academia staff in the Universiti Teknikal Malaysia Melaka (UTeM). His research interests are power electronics and drive, instrumentation, and DSP application.

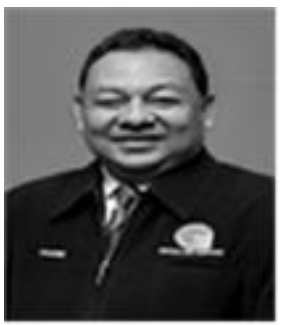

Mohd Rahimi bin Yusoff was born in Kelantan, Malaysia on 1967. He received his M.Sc. in Electrical Engineering from University of Nottingham, England in 1992 and B.Eng. in Electrical \& Electronics from University of Wales, Swansea, UK in 1990. Since 2010, he has been an academia staff in Universiti Teknikal Malaysia Melaka (UTeM) and currently holding the post as Dean of Faculty of Engineering Technology. His research interests are power quality, signal processing, and DSP application. 